\title{
Proyecto Técnico Ecourbano apoyado en las TIC para el aprendizaje STEM (Dibujo Técnico) y la consolidación de los ODS en el aula.
}

\section{Ecourban Technical Project supported by ICT for STEM learning (Technical Drawing) and the consolidation of SDG in classroom}

\author{
Francisco del Cerro Velázquez. \\ Universidad de Murcia. Murcia, España \\ fcerro@um.es \\ Fernando Lozano Rivas. \\ Universidad de Murcia. Murcia, España \\ fernando.lozano@um.es
}

\begin{abstract}
Resumen
El objetivo del presente artículo es presentar los resultados de aprendizaje con estudiantes de Dibujo Técnico en primer curso de bachillerato, como consecuencia de la realización de un Proyecto Técnico Ecourbano apoyado en las TIC. A lo largo del proceso, los participantes han desarrollado competencias descritas en la LOMCE tales como el fomento de la creatividad, el pensamiento crítico, el uso de las tecnologías de la información y la comunicación (TIC) en el aula y han puesto en valor los Objetivos de Desarrollo Sostenible (ODS), trabajando los contenidos establecidos en el decreto curricular tales como geometría plana, croquización y sistemas de representación. Mediante un método cuantitativo experimental, la investigación muestra resultados que evidencian una mejora significativa tanto en la motivación como en el aprendizaje del alumnado en el área. La experiencia ha permitido comparar el rendimiento académico de los alumnos que participaron en el proyecto frente al resto, evidenciando una mejora notable de los primeros en sus calificaciones a finales de curso y, por tanto, ha posibilitado considerar la potencialidad del proyecto técnico ecourbano como medio de aprendizaje e instrumento de evaluación en el área de Dibujo Técnico.
\end{abstract}

Palabras clave

Proyecto técnico, motivación, TIC, Dibujo Técnico, ecourbanismo, STEM.

\begin{abstract}
The aim of this article is to present the learning results with Technical Drawing students in the first year of high school, as a consequence of the realization of an Ecourban Technical Project supported by ICT. Throughout the process, the participants have developed skills described in the LOMCE such as the promotion of creativity, critical thinking, the use of ICT in the classroom and have valued the Sustainable Development Goals (SDG), working the contents established in the curricular decree such as flat geometry, croquización and systems of representation. Using an experimental quantitative method, the research shows results that evidence a significant improvement in both the motivation and learning of students in the area. The experience has made it possible to compare the academic performance of the students who participated in the project with the rest, evidencing a notable improvement of the former in their grades at the end of the year and, therefore, has made it possible to consider the potential of the ecourban technical project as a means of learning and an evaluation instrument in the area of Technical Drawing
\end{abstract}

\section{Keywords}

Technical Project, motivation, ICT, Technical Drawing, Ecourbanism, STEM. 


\section{Introducción}

Una de las metas que se espera alcance el alumnado de bachillerato al terminar la etapa es que sean capaces de resolver exigencias presentes y futuras. El dibujo técnico dota a los estudiantes de competencias para poder comunicarse, expresar ideas creativas, crear proyectos técnicos mediante croquis, bocetos y construcciones gráficas. Por tanto, esta materia es esencial como medio de comunicación en cualquier proceso de investigación o proyecto que se desee diseñar.

Tal y como establece la Ley Orgánica 8/2013, de 9 de diciembre, para la mejora de la calidad educativa:

Las habilidades cognitivas, siendo imprescindibles, no son suficientes; es necesario adquirir desde edades tempranas competencias transversales, como el pensamiento crítico, la gestión de la diversidad, la creatividad o la capacidad de comunicar, y actitudes clave como la confianza individual, el entusiasmo, la constancia y la aceptación del cambio.

A lo largo del segundo curso de bachillerato, el currículo establece un nuevo bloque de contenidos denominado proyecto, que tiene como finalidad que el alumnado vincule los contenidos y destrezas adquiridos en el área a lo largo de la etapa y las materialice en el diseño de un proyecto de tipo industrial o arquitectónico, enfatizando en la importancia que tienen la inclusión de las herramientas CAD para que el alumno alcance una percepción más universal de la realidad a la hora de proyectar. La presente investigación analiza la importancia de este aprendizaje desde la perspectiva innovadora del ecourbanismo, entendiéndolo como la "planeación y gestión de entornos urbanos teniendo en cuenta todos los criterios medioambientales que implica la ocupación de una comunidad con el fin de gestionar áreas urbanizadas sostenibles" (Arkiplus, 2017), a través de las TIC, es decir, partiendo de la contextualización actual del alumnado, desde su entorno urbano más próximo. En este sentido, se ofrece una nueva oportunidad de aprender apostando por la ciudad como nicho de aprendizaje. Una ciudad se puede estudiar desde infinitos ángulos; desde la historia, la economía, la política, el arte, la ingeniería y arquitectura. Y realmente, éstas no serían las únicas perspectivas posibles, porque la ciudad, "la más comprensiva de las obras del hombre lo reúne todo, y nada que se refiera al hombre le es ajeno" (Chueca, 2009).

La asignatura de dibujo técnico no debe entenderse solamente como una oportunidad de adquirir contenidos, es algo mucho más profundo, es crear en el alumno incentivos para, observando su entorno y, su realidad, éste sea capaz de analizarla, plantear planes de mejora para modificarla, mejorarla y de esa forma llegar a proyectar nuevos espacios de oportunidad que enriquezcan el bienestar de sus semejantes.

Podríamos afirmar que transformando su realidad mediante proyectos técnicos ecourbanos en el área de dibujo técnico, el alumno es capaz no solamente de crear, sino de alcanzar competencias que permitan la inclusión de los Objetivos de Desarrollo Sostenible a lo largo de su formación. Los ODS se plantean como una lista de tareas pendientes de la humanidad para un planeta sostenible, una hoja de ruta clara para un futuro mejor. El ecourbanismo, a través del proyecto técnico, ofrece al alumnado una oportunidad para expresar, compartir ideas y de esa manera poder tomar decisiones para fomentar espacios sostenibles en su entorno Naciones Unidas (2019):

La educación es la base para mejorar nuestra vida y el desarrollo sostenible. Además de mejorar la calidad de vida de las personas, el acceso a la educación inclusiva y equitativa puede ayudar abastecer a la población local con las herramientas necesarias para desarrollar soluciones innovadoras a los problemas más grandes del mundo.

En este artículo se valora la mejora del rendimiento que el método de proyectos a través del ecourbanismo apoyado en las TIC proporciona en el área de Dibujo Técnico para estudiantes de bachillerato, se propone la metodología de trabajo PBL (Project based learning) mediante un proyecto urbanístico integral que realizaron los alumnos sobre su entorno y que les permitió, por un lado, que los mismos alcanzaran las competencias establecidas en el Decreto Curricular de bachillerato, unos mejores resultados académicos en el proceso de evaluación frente a los alumnos de su mismo curso que no realizaron el proyecto innovador y, por último, proporcionó al profesor la oportunidad de trabajar en el aula la mayoría de los estándares relacionados con los tres bloques de contenidos: Geometría y dibujo técnico, sistemas de representación y normalización. Esta metodología basada en proyectos ha tenido una gran acogida en diferentes ámbitos educativos, y sus enfoques se han implementado en diferentes contextos propedeúticos internacionales (Ausin, Abella, Delgado y Hortigüela, 2016). Trabajar mediante el PBL permite al 
alumnado tomar conciencia y responsabilidad de su propio aprendizaje y "descubren sus preferencias y estrategias en el proceso" (García-Valcárcel y Basilotta, 2017).

\section{Importancia del ecourbanismo apoyado en las TIC en la enseñanza STEM: Caso del Dibujo Técnico.}

La medición de la contribución de las TIC al desarrollo ha sido una de las principales preocupaciones de distintas organizaciones y asociaciones en los últimos años. Según la UNESCO, las Tecnologías de la Información y la Comunicación tienen el potencial de ampliar en forma significativa las oportunidades de aprendizaje al alcance de diversas poblaciones y representan un medio que permite a los docentes y formuladores de políticas educativas mejorar tanto la calidad del proceso de enseñanza y aprendizaje como los logros educativos. A través del uso innovador de estas tecnologías, los centros educativos pueden contribuir a impulsar el desarrollo de aptitudes requeridas por la sociedad de la información (UNESCO, 2009).

Tal es así, que esta organización considera necesario aprovecharlas para poder alcanzar las metas en educación 2030; en definitiva, sacar partido del potencial de las TIC para lograr el ODS 4 en el año horizonte. A tal efecto del 23 al 25 de mayo del año 2015 en la ciudad china de Qingdao tiene lugar la Conferencia Mundial sobre las TIC y la Educación después de 2015, en cuyo informe (UNESCO, 2015) se afirma que "La utilización de las TIC en la educación mejora el intercambio de conocimientos, la recopilación de datos, la calidad del aprendizaje y la eficacia de los servicios".

Dicha conferencia fue el inicio del proyecto iniciado por la UNESCO "Aprovechar las TIC para alcanzar las metas de Educación 2030", proyecto conjunto del Fondo Fiduciario UNESCO-Grupo Weidong que durante cuatro años ayudaría a los Estados Miembros participantes a sacar partido del potencial de las TIC para lograr el ODS 4 hasta el año 2030. Dicho proyecto incluiría llevar a cabo el foro mundial sobre educación y TIC, mediante diversas actividades tales como: la creación de portales web de Recursos Educativos Abiertos, organización de una red internacional sobre las TIC en educación 2030 (INIE, 2030) y directrices políticas y carpetas didácticas sobre el uso de las TIC en educación (UNESCO, 2018).

Es en el año 2017 cuando la UNESCO publica el comunicado de Qingdao, estrategias de movilización de las TIC para realizar la agenda Educación 2030, en el cual establecen bases directamente vinculadas con UNESCO (2017):

- $\quad$ Prioridad para el acceso y el uso equitativos de las TIC.

- Políticas destinadas a explotar las TIC en pro de la calidad y la pertinencia del aprendizaje.

- Soluciones integradas de TIC en todos los ámbitos del ODS 4 y resto de ODS.

- $\quad$ Promoción del desarrollo de soluciones de TIC para la educación sostenidas en el plano local.

- Mecanismos de coordinación para fortalecer la cooperación regional e internacional.

El conocimiento de la ciencia y en nuestro caso de las tecnologías contribuyen significativamente en la vida personal, social y profesional de las personas y, por tanto, su comprensión es fundamental para la "preparación de la vida" de una persona joven OCDE (2017).

En nuestro ámbito nacional, conforme han pasado los años, las distintas leyes de educación han ido modificando los contenidos del área de dibujo técnico y expresión plástica y poco a poco se han ido implementando las TIC como instrumento indispensable para que el alumnado alcance las competencias establecidas y concretadas por los decretos curriculares, hasta llegar a un punto como es el caso de la ley actual de educación española en la que se establece lo siguiente LOMCE (2013):

La incorporación generalizada al sistema educativo de las Tecnologías de la Información y la Comunicación (TIC), que tendrán en cuenta los principios de diseño para todas las personas y accesibilidad universal, permitirá personalizar la educación y adaptarla a las necesidades y al ritmo de cada alumno o alumna $[\ldots]$ serán una pieza fundamental para producir el cambio metodológico que lleve a conseguir el objetivo de mejora de la calidad educativa.

Tal y como afirman Colás, De Pablos y Ballesta (2018): 
La incidencia de las TIC en la enseñanza en el sistema educativo español, los datos obtenidos y presentados a lo largo de este texto ponen en evidencia que las TIC actúan como un factor dinamizador de los procesos de enseñanza-aprendizaje, propiciando transformaciones respecto a los roles docentes-discentes. En este sentido, el aspecto más llamativo es que los roles de los profesores y alumnos se orientan a trabajar de forma constructivista, en tanto se potencia que el profesor se convierta en facilitador y guía del proceso de enseñanza-aprendizaje, mientras que se propicia que el alumno tenga un papel más activo en su proceso de aprendizaje, así como una mayor autonomía y responsabilidad en los procesos de toma de decisiones, a la mediación de las TIC en los procesos de enseñanza y aprendizaje, y a los aprendizajes.

Si algo caracteriza al Siglo XXI relativo a la educación es la era tecnológica en la que toda la comunidad educativa está inmersa. Ya es complicado encontrar centros educativos por toda España en los cuales no exista un aula TIC así como pizarras digitales en las aulas. La tecnología llegó a los centros y ha decidido quedarse. Es por ello que los centros educativos se han ido adaptando a estos contextos Marqués (2012), modificando los currículos, adaptando contenidos, introduciendo contenidos digitales transversales a todas las áreas y poniendo en valor las TIC dentro del proceso de enseñanza- aprendizaje. Según Pisa y Novejarque (2017), las TIC aportan muchísimas ventajas en el centro educativo, siendo superiores a los inconvenientes ya que éstas permiten trabajar en un entorno común de trabajo, facilitan la aplicación de recursos que integran de una manera muy eficaz las herramientas digitales en la dinámica de las clases, consiguiendo una mejora en el rendimiento y aprendizaje del alumnado. Sin embargo, no sólo es necesaria la infraestructura e implantación de las TIC en las aulas (Cabero, 2010), se hace imprescindible unos materiales didácticos adecuados y accesibles a toda la comunidad educativa, así como una formación del profesorado. Por otro lado, se entiende necesaria la colaboración de los docentes para crear una verdadera cultura colaborativa de intercambio de información y de materiales entre profesores en definitiva, que los docentes también practiquemos el trabajo colaborativo.

Una base en educación plástica es necesaria en etapas tempranas para poder enfrentarse a la materia de Dibujo técnico con herramientas suficientes y poder obtener los resultados esperados. Autores como Gacto y Albadalejo (2014) establecen conclusiones a tal efecto y afirman que incluso habiendo recibido formación en expresión gráfica o plástica durante su etapa en la ESO, se evidencian ciertas dificultades en estos alumnos a la hora de resolver problemas de tipo espacial, y entienden que es debido al mal entrenamiento de los mismos en etapas de educación anteriores y, por tanto, se considera que implementar las TIC en las primeras áreas de formación son importantes para que mejoren su capacidad espacial desde edades tempranas.

La materia de Dibujo Técnico es esencial para el alumnado que va a estudiar un grado de la rama científico tecnológica; grados como el de Arquitectura, Civil o Industriales son sólo algunos ejemplos de estudios superiores cuya carga lectiva en áreas vinculadas al Dibujo Técnico son más que evidentes; por tanto, una formación previa, sólida y bien planteada es necesaria en los dos cursos de bachillerato, previo al acceso de dichos alumnos a estos grados universitarios. Sin embargo, tal y como afirman Navarro, Saorín, Contero y Conesa, (2004), en ocasiones ciertos docentes universitarios del área se encuentran con ciertas dificultades relacionadas con la formación de los alumnos que acceden a estos grados tales como - $\quad$ El área de Dibujo Técnico, debe incluir contenidos directamente relacionados con el diseño asistido por
ordenador.

Los alumnos pertenecen a la era tecnológica y en ocasiones se sienten defraudados al no cumplirse sus expectativas ya que las metodologías tradicionales les ofrecen contenidos visualmente no atractivos.

El uso de las TIC en el proceso de enseñanza aprendizaje es necesario a lo largo de la etapa de Secundaria, así queda establecido en la LOMCE. En el tema que nos ocupa, sí que podemos asegurar que el diseño asistido por ordenador CAD, es una herramienta imprescindible que permite al alumnado poder materializar sus propuestas en 3D aplicando técnicas digitales que van a enriquecer y a ajustar a la realidad deseada las estructuras y espacios proyectados por ellos y, de esta forma, consolidar todo lo aprendido. Sin duda las TIC son herramientas de Vanguardia en STEM y especialmente en Dibujo Técnico. El uso de programas y aplicaciones 3D en el diseño y resolución de casos prácticos en el ordenador ayuda a desarrollar la visión espacial. Según distintos autores Batchelor y Wiebe (1995) si lo que pretendemos es alcanzar un mayor nivel en la capacidad espacial, se hace inevitable trabajar en el aula con modelos 3D que podamos manipular, voltear y sobre los que podamos realizar distintos diseños.

Por otro lado, el uso de estas tecnologías fomenta la creatividad en el área. Según Brittain y Lowenfeld, (2008): 
El desarrollo de la sensibilidad perceptiva que proporciona la creatividad debe pues convertirse en una parte primordial del proceso educativo, en la medida en que contribuye a generar seres humanos flexibles y creativos, dotados de recursos internos, capaces en definitiva de adaptarse de manera libre y responsable a la vida en sociedad. Afirman que dibujar es una aptitud relacionada con la conducta individual y las estructuras cognitivas.

Los recursos TIC que se encuentran a disposición de los docentes son muy variados y muy flexibles ya que se adaptan a todo tipo de materias; de ahí su transversalidad. Un ejemplo es la Realidad Aumentada. Este tipo de tecnología es muy adecuada en áreas STEM tales como el dibujo técnico y la expresión gráfica ya que gracias a ella, los alumnos pueden visualizar en 3D objetos y figuras en cuestión de segundos gracias a las aplicaciones actuales. Realizar estas construcciones a mano resultaría tedioso para ellos y se emplearía mucho tiempo de clase para poder llevar a cabo la tarea. La Realidad Aumentada mejora el aprendizaje de los contenidos, la visión espacial de los alumnos, así como el aprovechamiento del tiempo de clase Del Cerro y Morales (2017).

Tomando todo esto en cuenta, se hace necesaria una metodología en el área de Dibujo Técnico que permita a los alumnos desarrollar los contenidos curriculares de una manera activa, dinámica que les ofrezca retos que fomenten su creatividad, dinamismo y trabajo en equipo. Sin embargo, se estima necesario que el alumnado impulse valores que le haga entender la importancia de alcanzar esos objetivos globales que protejan al planeta y aseguren la prosperidad y bienestar para todos. Por ello, se considera el ecourbanismo a través del proyecto técnico perfeccionado por las TIC, una metodología significativa en estas edades, que centrándose en los contenidos, implique a los alumnos en actividades de investigación para resolver situaciones de la vida real mediante proyectos técnicos sostenibles.

En un estudio anterior (Del Cerro y Lozano, 2018) se concluyó que:

El ecourbanismo llevado al aula nos ha permitido desarrollar una metodología de trabajo cuyo resultado ha sido entre otros, un aumento en las capacidades del alumno. Por otro lado, el carácter instrumental del Dibujo Técnico ha permitido a los alumnos trabajar la interdisciplinariedad, la creatividad, la iniciativa, el trabajo en equipo y el pensamiento crítico, aptitudes puestas en valor en el currículo (Decreto 221/2015, de 2 de septiembre).

Por tanto, se nos plantea un reto; trabajar los ODS a través del ecourbanismo planteándoles a los alumnos una tarea mediante la siguiente reflexión. ¿Nuestro centro educativo es abierto, accesible, sostenible y nos ofrece áreas de convivencia de usos múltiples? El aula deja de ser un espacio exclusivo de las habilidades puramente cognitivas para convertirse en un entorno de trabajo donde los protagonistas van a emprender una nueva tarea educativa integral.

Santos, Almeida, Bragança y Barbosa (2019) afirman que el siglo XXI es el siglo del desarrollo sostenible y, por tanto,

La educación para la sostenibilidad en las escuelas secundarias tiene el potencial de hacer que los beneficios de la construcción civil sean más visibles para la sociedad y los medios de comunicación al mostrar a los estudiantes, padres y comunidades en general cómo la sostenibilidad en el entorno construido puede mejorar sus vidas en aspectos económicos, sociales y ambientales.

La escuela nueva no sólo transforma la forma de enseñar, sino que requiere nuevos espacios para facilitar la adquisición del conocimiento. En este contexto, el estudio de la arquitectura de cuatro metodologías de la escuela nueva localizadas en Cali (método Waldorf, método Montessori, metodología Etievan y método del Colegio Ideas) permite ver cómo la arquitectura puede contribuir en la consolidación de procesos educativos innovadores (Jiménez, 2009).

¿Qué papel juegan las TIC en todo esto?

La geometría, así como los sistemas de representación, son contenidos complicados de enseñar ya que el alumno debe no sólo comprender la fundamentación teórica en sí, sino poseer un razonamiento espacial adecuado a la hora de interpretar construcciones en distintos sistemas, como el diédrico o planos acotados.

Por ello, para proyectar y diseñar espacios, los programas y aplicaciones CAD nos ofrecen grandes bondades; desde una mayor precisión hasta mejorar la creatividad y diseño mediante modelos 3D. Tradicionalmente se ha enseñado geometría plana en los centros educativos mediante el trazado en la pizarra con tiza utilizando escuadras, cartabones, reglas y compases. Este tipo de metodologías no 
permiten agilizar el ritmo de la clase pues las construcciones elaboradas con este tipo de instrumentos ralentizan en ocasiones las explicaciones de las clases y entorpecen la visión de la explicación por parte del alumno ya que normalmente el profesor se interpone entre el trazado y el alumno a la hora de la explicación. El uso de las SmartBoard y programas CAD, ofrecen ventajas muy notables al respecto; son ágiles si el docente tiene un domino suficiente del software y se proyectan directamente en la pizarra sin obstáculos que entorpezcan la visión. Este tipo de programas permiten de una manera directa e unívoca simular construcciones tradicionales, ofreciendo movimientos, giros, cambios de plano de las mismas, en definitiva una presentación dinámica de los cuerpos geométricos.

¿Podríamos afirmar que un proyecto técnico ecourbano implementado en el aula mediante la metodología PBL a través de las TIC, es una herramienta didáctica que permitiría una notable mejora en la motivación y aprendizaje de los alumnos de bachillerato en el área de Dibujo Técnico?

\section{Objetivos de la investigación}

La finalidad última de esta investigación es analizar si el ecourbanismo a través de proyectos técnicos apoyados en las TIC, e implementados en el aula con alumnos de bachillerato de la asignatura de Dibujo Técnico, es un instrumento metodológico que ofrece una mejora significativa tanto en la motivación como en el aprendizaje de los alumnos en el área. Para ello, se plantean los siguientes objetivos específicos:

- Estudiar la potencialidad del proyecto técnico ecourbano como medio de aprendizaje e instrumento de evaluación en los niveles de bachillerato.

- Poner en valor la metodología PBL a través de las TIC como medio de trabajo en el aula de Dibujo Técnico.

- Considerar si esta metodología de trabajo fomenta la motivación de los alumnos.

\section{Metodología}

\subsection{Fase de muestro}

Esta experiencia docente se llevó a cabo con dos grupos de alumnos de $1 .^{\circ}$ de Bachillerato que eligieron voluntariamente la asignatura de Dibujo Técnico, impartida durante cuatro horas semanales. El número total de alumnos matriculados era 23. Para poder seleccionar a los candidatos sometidos a estudio que realizaron el proyecto se utilizó una estrategia de muestreo aleatorio simple, siendo la muestra total real de 9 candidatos. Por tanto, de los 23 alumnos matriculados, 9 realizaron el proyecto dirigidos por su profesor. Ambos grupos estaban formados por alumnos de características similares.

\subsection{Instrumentos y materiales}

Para poder realizar la experiencia, los alumnos emplearon materiales de diseño en la fase previa y así dibujar bocetos y planos en las que plasmar sus primeras ideas. En la fase de proyecto se apoyaron en herramientas CAD para proyectar en 3D los diseños realizados con los instrumentos de trabajo y, por último, materiales manipulativos para poder crear la maqueta del diseño final.

\subsection{Proyecto técnico ecourbano}

Mediante este proyecto técnico desarrollado con alumnos de $1 .^{\circ}$ de bachillerato se pusieron en valor las indicaciones establecidas en el Decreto curricular mediante la metodología de proyectos. En primer lugar, se les planteó a los alumnos un reto o problema al cual deben darle solución; proyectar un espacio de oportunidad, sostenible en el centro escolar que fomentara las relaciones sociales y las condiciones del entorno dando solución a los problemas detectados; espacios no accesibles, zonas inutilizadas, escasez de zonas verdes entre otras. Para iniciar el proyecto se les explicó que un espacio de oportunidad en el ámbito de la Ingeniería Civil y Arquitectura era un entorno o espacio que muestra posibilidades de transformación de un conjunto habitacional existente en el que se puede realizar una intervención mediante diferentes fases (Chacón, Valero y Valverde, 2012); continuando con un análisis previo de la situación o realidad que les circuncidaba.

"Un espacio bien planteado facilita y activa la labor docente y el aprendizaje, sin olvidar que el elemento clave en este proceso es el humano", Martínez y Ruíz (2014). Mediante el aprendizaje basado en proyectos, los alumnos abordaron los contenidos de la asignatura de una manera dinámica y práctica, 
activando el aprendizaje colaborativo partiendo de los conocimientos previos de los mismos. Las estrategias PBL son apropiadas para desarrollar capacidades tales como trabajar en equipo, fomentar el trabajo autónomo, planificar los tiempos. Según Alcober, Ruíz y Valero (2003):

Mientras tradicionalmente primero se expone la información y posteriormente se busca su aplicación en la resolución de un problema, en el caso del PBL primero se presenta el problema, se identifican las necesidades de aprendizaje, se busca la información necesaria y finalmente se regresa al problema.

Hertzberger (2008), afirma que uno de los principios en los que se ha basado el diseño de los centros educativos a lo largo del tiempo se centra en largos pasillos que unen espacios autónomos que no ofrecen posibilidades para aprender de diferentes formas. Es a partir de la segunda mitad del siglo XX cuando comienzan a vislumbrarse nuevas ideas en la creación de espacios de convivencia en el centro.

A los alumnos se les propuso un reto; estudiar las instalaciones del centro y encontrar espacios de oportunidad que fomentaran la convivencia en el mismo. Mediante este proyecto, nació una vía para dar respuesta a esas necesidades que tienen los propios alumnos, mejorar alguna instalación del colegio y, por tanto, su funcionalidad. Se planteó una iniciativa innovadora que aportara a esa arquitectura y urbanismo entendidos como estándar del centro, una nueva visión eco, tal y como establecen los Objetivos de Desarrollo Sostenible. Un proyecto que integraría, zonas de descanso, fomento de las relaciones sociales, ocio y zonas verdes, poniendo en valor los problemas ambientales en los que nos encontramos utilizando materiales ecológicos y usando energías verdes.

Los alumnos idearon, en primer lugar, distintas soluciones al problema y mediante un brainstorming analizaron los diferentes espacios de oportunidad en el colegio. Para ello, examinaron los planos de que disponían y realizaron un estudio exhaustivo de los espacios de todo el colegio elaborando nuevos croquis y planos. En la figura 1 se observan dos plantas de estado actual del centro a escala. En la figura 2, los alumnos comienzan a proyectar los espacios actuales para poder realizar el estudio funcional.
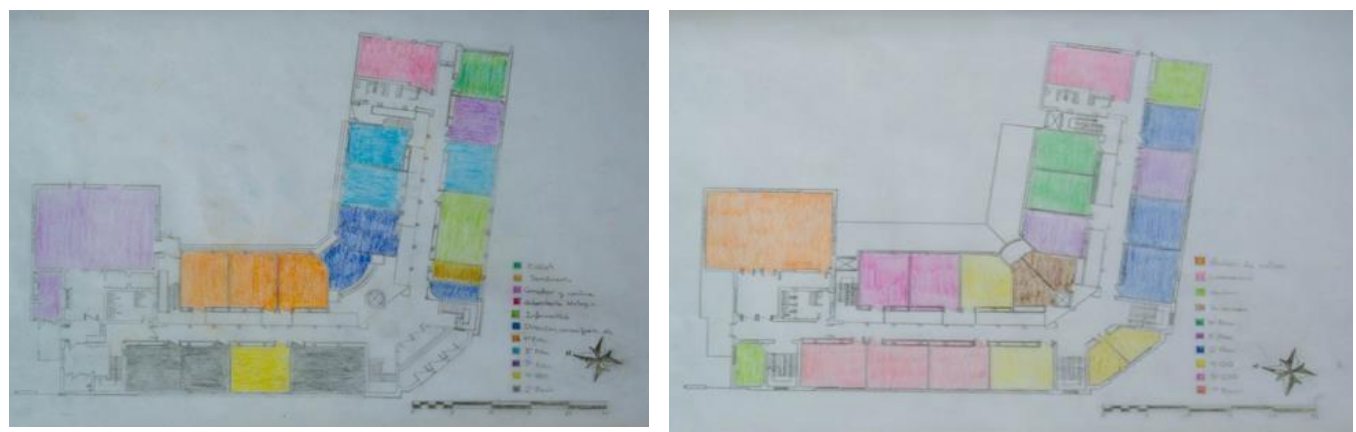

Figura 1: Diseño en planta de las aulas del centro.
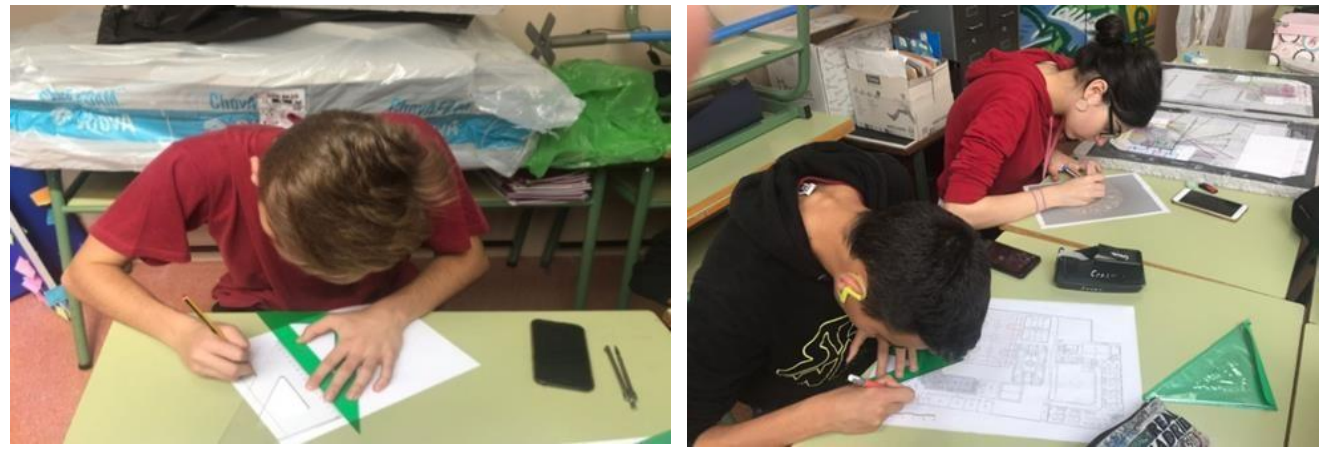

Figura 2: Alumnos participantes diseñando y acotando las plantas geométricas del centro. 
En la figura 3, observamos la planta del pabellón deportivo del colegio, posible zona de actuación.

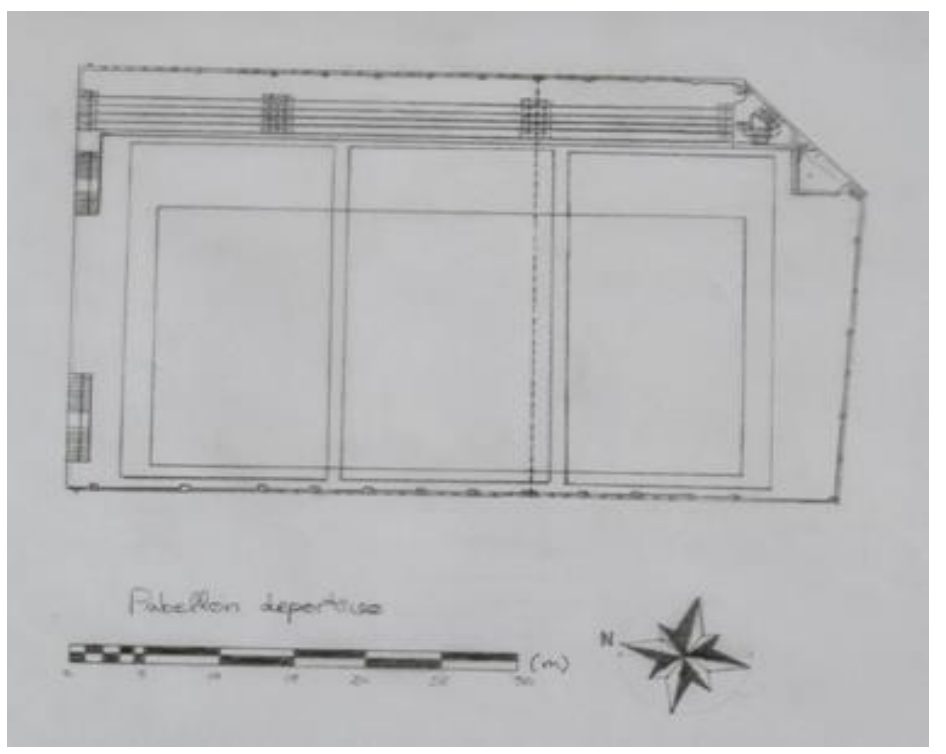

Figura 3: Diseño en planta, cotas y escala gráfica del pabellón deportivo del centro.

Se realizaron los estudios previos diseñando zonas exteriores del colegio que pudieran dar solución al problema planteado, poniendo en práctica los contenidos previamente explicados en clase, tales como representar formas tridimensionales sencillas a partir de perspectivas, fotografías, piezas reales o espacios del entorno próximo. En la figura 4 comprobamos el primer diseño en perspectiva de una zona del patio actual. Los alumnos estudiaron minuciosamente los espacios tales como pistas deportivas, pérgolas actuales y áreas de tránsito.

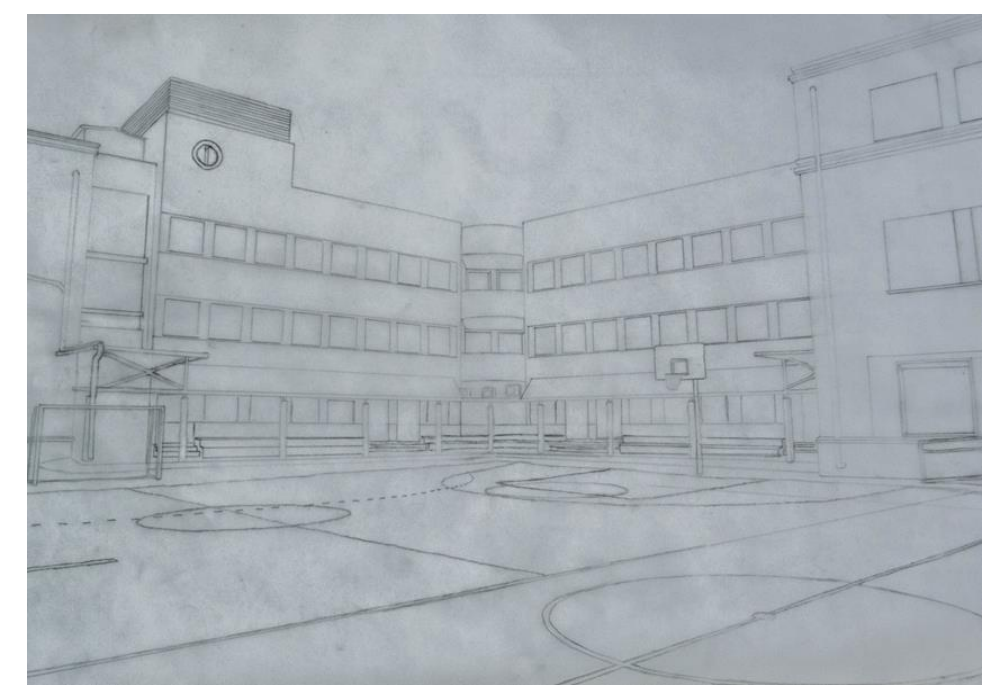

Figura 4: Diseño en perspectiva del patio del centro educativo

De manera paralela al estudio grafico de las posibles zonas de actuación, se planteó la necesidad de averiguar cuáles eran las zonas más transitadas por los alumnos y cuales las menos, para de esa forma poder reactivarlas y buscar el mejor espacio de oportunidad. Para ello, los alumnos sugirieron diseñar diagramas de flujo y, de forma muy creativa, fabricaron unas maquetas mediante hilos de color que les permitieran determinar las zonas menos transitadas, para en una etapa posterior del proyecto plantear soluciones viables y ecológicas que solventaran el problema. En la figura 5 los alumnos discuten cómo materializar los diagramas de flujo y comienzan a trabajar sobre las maquetas. 

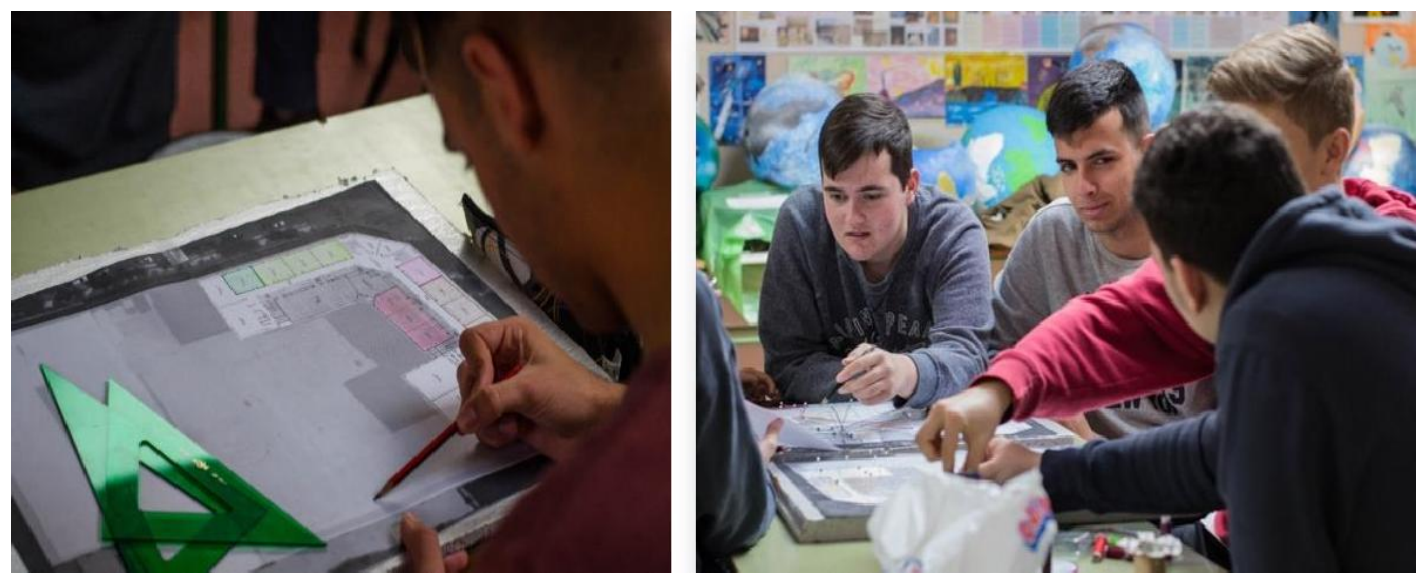

Figura 5: Diseño de los diagramas de flujos. Trabajo colaborativo

En esta fase del proyecto, se trabajaron en profundidad la croquización, las escalas, elementos geométricos básicos de la geometría plana, perspectivas y formas tridimensionales sencillas.

Mediante hilos de colores y coloreando las aulas del mismo color visualizaron los recorridos que hacen los alumnos en los tiempos de descanso.

Tras la primera fase de estudios previos, los alumnos obtuvieron las siguientes conclusiones:

- Consiguen determinar la zona de actuación menos transitada por los alumnos.

- Determinan un área amplia que les permitirá diseñar y proyectar sus ideas.

- Actuar en esa zona, implicaría eliminar la pista deportiva menos utilizada por ellos.

- Ofrecen zonas de descanso para esos alumnos que se sientan a diario en el suelo y se apoyan en el muro.

- $\quad$ Eligen una zona con gran soleamiento natural.

- Esta zona les permitiría reubicar la grada actual, situada junto a las aulas de infantil, impidiendo dar la clase en estos niveles de manera adecuada.

- El espacio de oportunidad elegido permite proyectar zonas de relación, descanso y zonas verdes.

En la segunda fase del proyecto, los alumnos determinan los elementos urbanísticos y arquitectónicos que van a diseñar para dar solución al estudio previo planteado, para ello, proyectaron una grada funcional y ergonómica que permitiría tanto el descanso como el juego. Se ejecutaría mediante hormigón autocompactable y estaría inspirada en las olas del mar. Conforme fueron madurando la idea, variaron ligeramente la concepción inicial. Las gradas les permitirían esconderse, descansar, fomentar las relaciones sociales entre ellos, siendo un espacio ideal para la lectura relajada así como para la tertulia.

- Concepción de la idea: Grada ergonómica. Mediante esta proyección los alumnos trabajaron elementos curvos, enlaces y tangencias tanto en planta como en alzado partiendo de sus ideas y conocimientos previos. Relacionaron este diseño con elementos naturales y ecológicos de su entorno; las olas del mar. En las figuras 6,7,8 y 9 se observan los primeros diseños en los que se inspiraron los alumnos. Podemos observar la evolución de sus ideas y la transición de la onda que comenzó siendo totalmente curva y que decidieron moldearla para favorecer espacios donde poder tumbarse para poder descansar, leer o simplemente relajarse. Estos dibujos fueron el origen de la futura grada proyectada. 

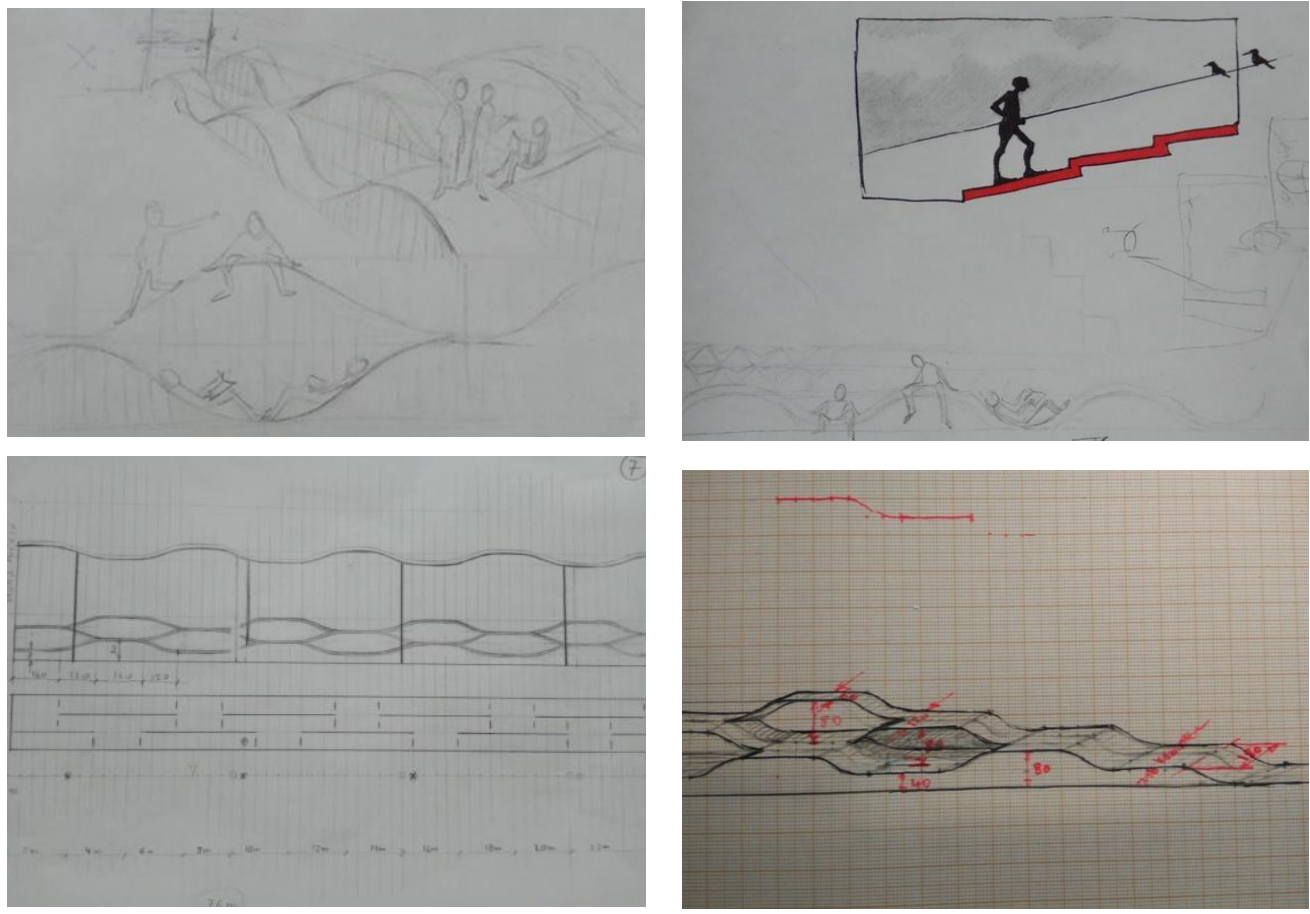

Figuras 6,7,8 y 9: Diseño de primeras ideas de la grada inspiradas por los participantes

- Proyecto en planta de la zona de ocio. Desarrollaron una zona de juegos inspirada en la naturaleza. Les parecido original plantear juegos clásicos en el suelo: diseñaron una Oca con forma de amonite y un damero. Junto a estos juegos, árboles y parterres con tierra vegetal ofrecían un entorno ecológico y agradable. Como pavimento, utilizaron caucho ecológico para la zona de juegos siendo el resto hormigón en masa. Mediante esta proyección, los alumnos trabajaron la creatividad y la capacidad de comunicar y expresar sus inquietudes. La figura 9 y 10 muestran los dibujos creados por los alumnos para la zona de juegos y un plano en planta con la distribución de las instalaciones junto a la pista deportiva, en la esquina proyectada se observan en planta los juegos, árboles y grada ergonómica.
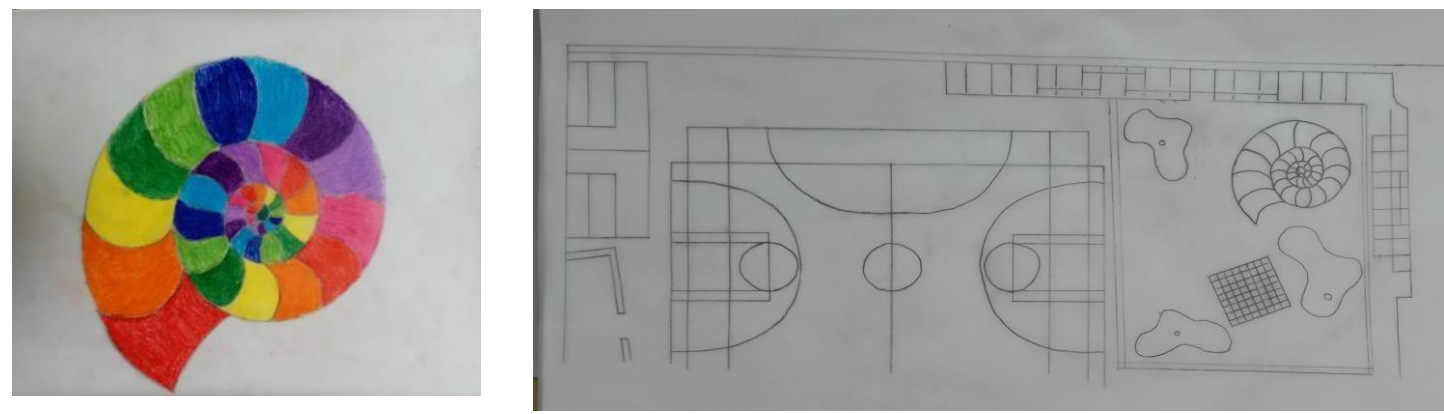

Figuras 9 y 10: Diseño de las zonas de juegos.

Alrededor de la zona proyectada colocaron un imbornal para recoger el agua de lluvia que por escorrentía y, tal como están las pendientes discurre en la zona. Pensaron en un principio colocar pérgolas para ofrecer zonas de sombra, pero finalmente entendieron que el ecourbanismo es importante a la hora de proyectar y decidieron colocar cables de acero y suspendidos de los mismos, tejidos fotovoltaicos para aprovechar la luz solar, tan presente en la zona de Cartagena. Los cables de acero les permitirían de la misma forma, colgar sus trabajos como puntos de exposición.

La forma triangular de los tejidos fotovoltaicos se inspiró como la grada, en el ambiente marino simulando velas de barcos. Mediante esta proyección, los alumnos trabajan triángulos, croquización, escalas y perspectivas. Las figuras 11,12 y 13 muestran los diseños de los tejidos unidos mediante cables de acero que ofrecerían sombra a la zona proyectada. La figura 13 representa el diseño en perspectiva de 
la zona proyectada ofreciendo una idea global del espacio de convivencia; grada ergonómica, zona de juegos, zonas verdes y espacios de descanso.
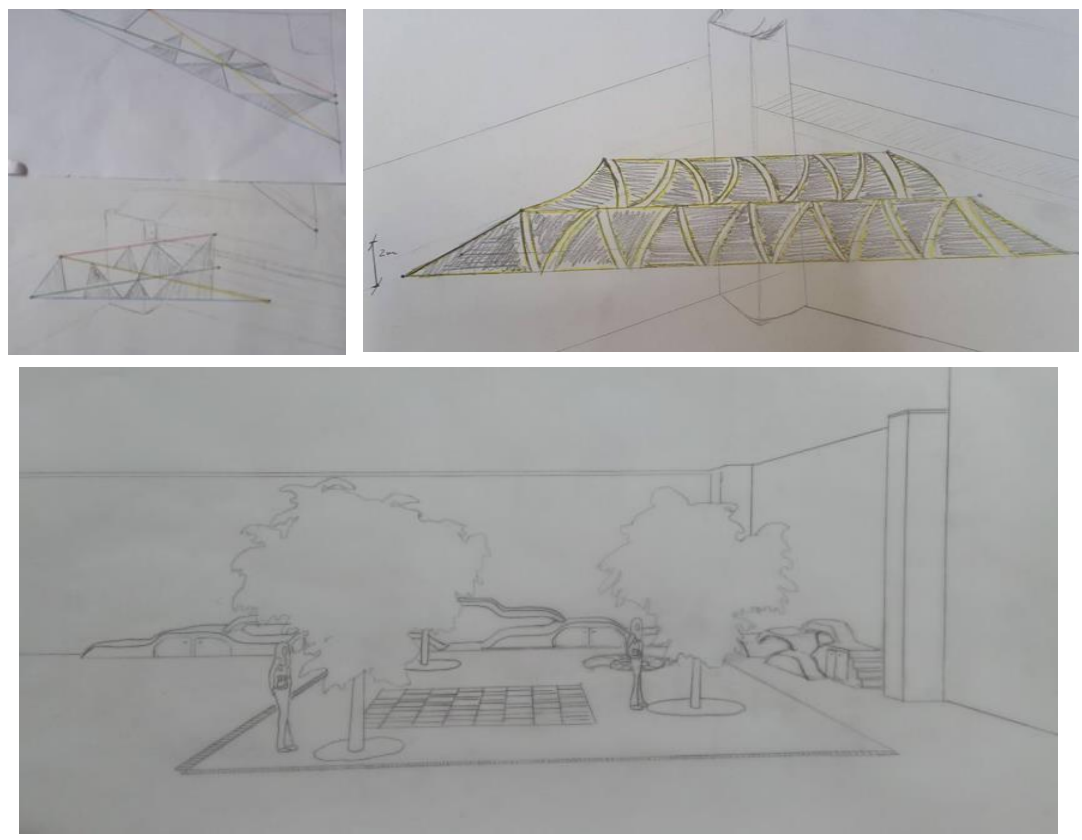

Figuras 11,12 y 13: Diseño de paneles fotovotaicos y 3D del espacio de oportunidad.

Las TIC en el área de dibujo técnico son esenciales para mejorar entre otras cosas la percepción visual de los alumnos. En esta era tecnológica en la que la inmersión de las TIC es más que evidente, se estimó necesaria la implantación de las mismas en el proyecto.

De esta forma, los alumnos emplearon aplicaciones CAD para el diseño final del proyecto y de esta forma enriquecer su competencia digital. Utilizaron programas tales como GIMP para retocar digitalmente las fotografías empleadas, Sketchup para el diseño en 3D de la zona de actuación, portales como Goolzoom Mapas con el que trabajaron la cartografía en planta, cotas, escalas y perfiles longitudinales del terreno, así como, la elaboración de los planos de situación, emplazamiento y planta de estado actual. Por ultimo, utilizaron el geoportal Urbanismo Cartagena con el que afianzaron sus conocimientos sobre ortofotos, imágenes satelite y cartografía catastral. Las figuras 14 y 15 muestran a los alumnos en el aula empleando los softwares antes mencionados. Las figuras 16, 17 y 18 ofrecen una visión más real del proyecto. En este proceso los alumnos pudieron desarrollar su competencia digital mejorando su capacidad espacial. En esta fase eligieron cromatismos y texturas fomentando su creatividad e imaginación. Las figuras 19 y 20 muestran el resultado final de la experiencia; distintas perspectivas del espacio de convivencia proyectado.
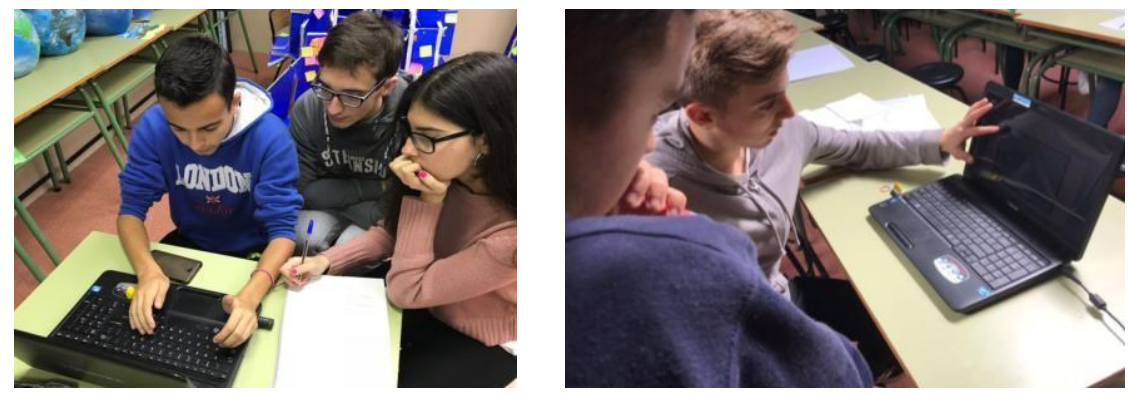

Figuras 14 y 15: Trabajo colaborativo TIC. 

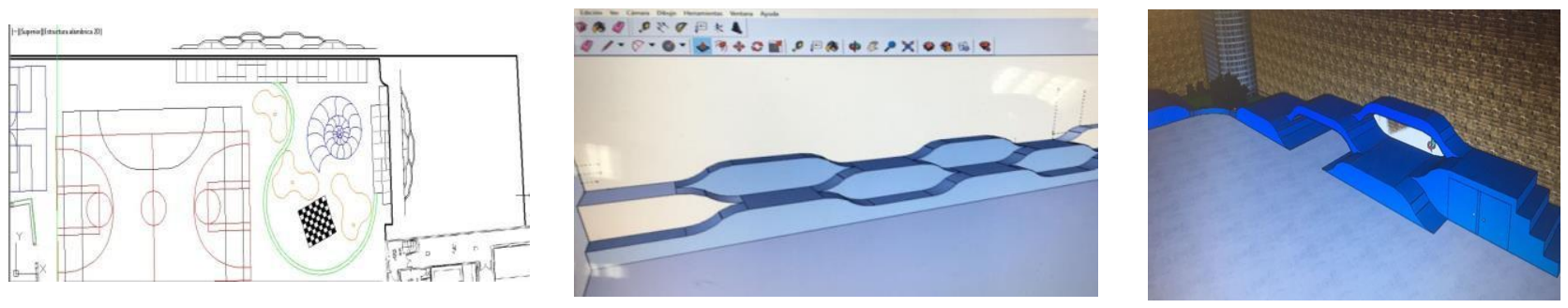

Figuras 16, 17 y 18: Primeros diseños 3D mediante programas CAD.
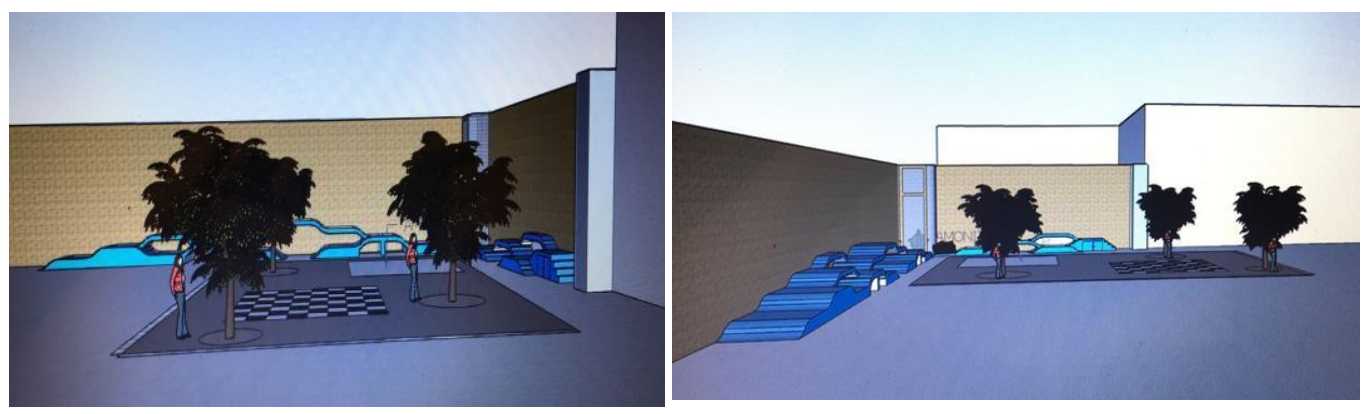

Figuras 19 y 20: Diseño final del espacio de oportunidad en 3D mediante programas CAD.

\subsection{Método y procedimiento}

En la investigación se siguió el paradigma empírico-analítico a través de un método cuantitativo experimental mediante un grupo de control formado por 14 alumnos y otro grupo experimental formado por 9 alumnos. El proyecto se realizó durante 12 semanas. A comienzo de curso (2017-2018) se realiza la evaluación inicial a los 23 alumnos mediante una prueba teórico-práctica de la que se obtiene que los 23 poseen un nivel adecuado y suficiente para poder desarrollar el curso con normalidad.

Tras la finalización del proyecto completadas las 12 semanas los alumnos realizaron la exposición y defensa pública del mismo en El Salón de Actos de la Escuela de Arquitectura de la Universidad Politécnica de Cartagena, donde el jurado del concurso les otorgó el primer premio.

Se procedió a la evaluación de la asignatura mediante una prueba global que recogía los contenidos principales del curso. Cuatro ejercicios prácticos; geometría plana, tangencias, sistema diédrico y, por último, perspectivas y vistas. Ambos grupos, tanto el de control como el experimental, realizaron la misma prueba siendo los criterios de evaluación los mismos para todos. Los resultados obtenidos por los alumnos de ambos grupos se analizan a continuación.

Para completar la investigación, se les pasó un cuestionario tipo Likert a los 9 participantes para medir el nivel de satisfacción, motivación y otros aspectos que permitan aportar datos a los resultados finales.

\subsection{Análisis de los resultados}

Para poder analizar los resultados, se utilizó la herramienta SPSS aplicándose la prueba U de Mannwhitney. Son dos muestras independientes con $\mathrm{N}<30$ siendo la variable factor "realizar o no el proyecto". Se analizaron los dos grupos mediante una variable dependiente: las calificaciones de ambos grupos; para ello empleamos un $\alpha=5 \%$, mediante dos hipótesis:

$\mathrm{H}_{0}$ : No existe una diferencia significativa entre la media de las calificaciones del grupo de control y la media de las calificaciones del grupo experimental. 
$\mathrm{H}_{1}$ : Existe una diferencia significativa entre la media de las calificaciones del grupo de control y la media de las calificaciones del grupo experimental.

La media de las calificaciones de la prueba final para cada uno de los grupos fue la siguiente:

Grupo "No hace el proyecto" = 5,29; Grupo "Hace el proyecto"=9,22.

Realizada la prueba y obtenidos los estadísticos de la misma, se comprobó un nivel de significación asintótica bilateral de .000 . Este valor era menor que $\alpha$ y, por tanto, se rechazó la hipótesis nula, pudiendo afirmar que la diferencia entre la media de las calificaciones entre ambos grupos era significativa. En la figura 21 puede observarse un gráfico que muestra la diferencia entre la media de las calificaciones de los alumnos del grupo de control frente al experimental.

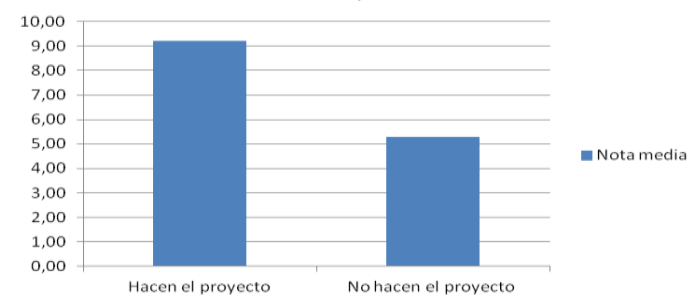

Fig 21: Diagrama de resultados. Comparación de medias.

Para analizar el grado de satisfacción de la experiencia, se les pasó un cuestionario tipo Likert a los alumnos que realizaron el proyecto con respuestas que variaban desde 1 (menor puntuación) hasta 5 (mayor puntuación). Los resultados se analizaron con el software SPSS tal y como se muestran a continuación en la tabla 1.

La escala se ha medido siguiente el modelo de respuesta que se presenta a continuación:

1- Totalmente en desacuerdo.

2- En desacuerdo.

3- Sin opinión definida.

4- De acuerdo.

5- Totalmente de acuerdo

Tabla 1. Resultados del cuestionario tipo Likert.

\begin{tabular}{|c|c|c|c|c|c|c|}
\hline & & $\begin{array}{l}\text { El Proyecto ha } \\
\text { fomentado la } \\
\text { creatividad e } \\
\text { iniciativa }\end{array}$ & $\begin{array}{l}\text { El proyecto ha } \\
\text { permitido y } \\
\text { mejorado el } \\
\text { trabajo en equipo }\end{array}$ & $\begin{array}{c}\text { El uso de las TIC } \\
\text { han sido } \\
\text { motivadoras }\end{array}$ & $\begin{array}{l}\text { Las herramientas } \\
\text { CAD han } \\
\text { facilitado el } \\
\text { aprendizaje }\end{array}$ & $\begin{array}{l}\text { La metodología } \\
\text { PBL ha sido } \\
\text { satisfactoria }\end{array}$ \\
\hline \multirow[t]{2}{*}{$\mathrm{N}$} & Válido & 9 & 9 & 9 & 9 & 9 \\
\hline & Perdidos & 14 & 14 & 14 & 14 & 14 \\
\hline \multicolumn{2}{|c|}{ Media } & 4,0000 & 4,0000 & 4,5556 & 4,5556 & 4,1111 \\
\hline \multicolumn{2}{|c|}{ Mediana } & 4,0000 & 4,0000 & 5,0000 & 5,0000 & 4,0000 \\
\hline \multicolumn{2}{|c|}{ Moda } & 4,00 & 4,00 & 5,00 & 5,00 & 4,00 \\
\hline \multicolumn{2}{|c|}{ Desviación estándar } &, 50000 & ,70711 &, 52705 &, 52705 & 60093 \\
\hline \multicolumn{2}{|c|}{ Rango } & 2,00 & 2,00 & 1,00 & 1,00 & 2,00 \\
\hline
\end{tabular}




\section{Discusión}

La LOMCE, marco legal entorno al cual pivota el sistema educativo español expone que a lo largo de la etapa educativa no sólo son necesarias las habilidades cognitivas de los alumnos sino que es imprescindible establecer nuevas metodologías de aprendizaje que les permitan adquirir competencias transversales relacionadas con el pensamiento crítico, creatividad o la capacidad de comunicar.

A través del Ecourbanismo como práctica innovadora en la enseñanza-aprendizaje de las materias STEM, se ha propuesto un proyecto sostenible aplicado en el área de Dibujo técnico apoyado en las TIC para alumnos de $1 .^{\circ}$ de bachillerato que nos ha permitido reforzar algunas de las aportaciones revisadas en este estudio de investigación.

Se corroboran las conclusiones recogidas por la UNESCO en la Conferencia Mundial sobre las TIC y la Educación después de 2015, ya que como muestran los resultados del presente estudio de investigación, las TIC implementadas en el proyecto han permitido una mejora en la calidad del aprendizaje de los alumnos y, por tanto, coincidimos también con Colás, De Pablos y Ballesta (2018) en que las TIC dinamizan y mejoran notablemente el proceso de enseñanza-aprendizaje. Por otro lado, se confirman las teorías de Ausin, Abella, Delgado y Hortigüela (2016) y García-Valcárcel y Basilotta (2017), ya que el alumnado ha acogido esta experiencia con ilusión y han trabajado con mucha motivación, responsabilizándose de su aprendizaje a lo largo de las fases del proyecto.

Los resultados manifiestan, por otro lado, que si bien la experiencia ha sido valorada por los alumnos como positiva, el fomento de la creatividad no ha sido la competencia mejor evaluada por ellos así como tampoco el fomento del trabajo en equipo. No obstante, si observamos la tabla de resultados, los 5 ítems encuestados tienen una valoración mínima de 4, es decir, podemos afirmar que en todos los casos los alumnos están muy satisfechos con la experiencia siendo importante destacar el resultado relacionado con la motivación y las TIC, siendo dicho valor de 4,5556. Por tanto el uso de las TIC y programas CAD en el desarrollo del proyecto ha motivado y facilitado el aprendizaje de los alumnos.

El nivel de significancia es relevante en el estudio de investigación. Los resultados muestran con claridad que los alumnos que participaron en el proyecto obtuvieron resultados académicos muy superiores frente a sus compañeros que no lo realizaron. Esta realidad nos hace reflexionar sobre la importancia que ha tenido esta implementación metodológica en el aula así como el aumento en el rendimiento académico de los alumnos. Por otro lado, estos resultados pueden aportar respuestas a las inquietudes contribuidas por Navarro, Saorín, Contero y Conesa (2004).

Mediante esta experiencia se han puesto en valor los ODS a través de contenidos curriculares y, por tanto, contribuimos a las estrategias de movilización del comunicado de Qingdao ya que, desde una perspectiva holística, entendemos que el desarrollo de este proyecto educativo ha apostado, entre otras, por la promoción del desarrollo de soluciones TIC para la educación sostenidas en el plano local.

Los alumnos participantes en el proyecto fueron los ganadores de la I Olimpiada de Arquitectura de la Región de Murcia en la que compitieron contra 40 equipos de 26 centros educativos (públicos, concertados y privados) que abarcaban hasta 15 municipios diferentes de la Región de Murcia y que supuso la implicación de 242 alumnos de secundaria y bachillerato.

Como sugerencia cabe mencionar que, en el caso de realizar futuras intervenciones similares en el aula, se consideraría positivo poder realizar esta experiencia con una muestra mayor y, de esa forma, poder comparar ese estudio con los resultados obtenidos en el presente.

\section{Conclusiones}

La presente investigación ha aportado la puesta en marcha de una propuesta educativa concreta en el área de Dibujo Técnico mediante la cual, alumnos de $1 .^{\circ}$ de Bachillerato han podido desarrollar los contenidos establecidos en el currículo actual mediante el ecourbanismo como instrumento metodológico a través de las TIC. Este proyecto técnico sostenible ha contribuido a la adquisición de competencias transversales establecidas en la LOMCE tales como la creatividad, la capacidad de comunicar y el trabajo en equipo, entre otras.

Se evidencia la potencialidad del proyecto técnico ecourbano como medio de aprendizaje e instrumento de evaluación en los niveles de bachillerato. Este trabajo ha permitido que los alumnos mejoren su rendimiento académico, proyectando y diseñando un espacio nuevo eco y sostenible desde sus propias 
ideas, buscando soluciones reales de su entorno que mejoren la calidad de vida de sus congéneres, empleando contenidos establecidos en el currículo, tanto en el campo de la geometría plana, como en los sistemas de representación. De la misma forma, esta iniciativa didáctica ha puesto en valor competencias transversales descritas en los ODS permitiendo que en los alumnos nazca el espíritu crítico y una actitud positiva con el Desarrollo Sostenible ya que nos ha permitido educar alumnos comprometidos con su entorno y con sus semejantes.

Mediante todo el desarrollo del proyecto se ha analizado el ecourbanismo como instrumento metodológico mediante un proyecto técnico apoyado en las TIC, implementado en el aula con alumnos de bachillerato de la asignatura de Dibujo Técnico obteniendo resultados positivos en el estudio. Una de las claves del éxito del proyecto ha sido el trabajo colaborativo. Los alumnos han trabajado en grupo aportando y debatiendo ideas, fomentando la comunicación como competencia transversal; expresando sus inquietudes, consensuando y buscando soluciones reales de su entorno que fomente la mejora en la vida de sus semejantes proyectando un espacio de convivencia eco y sostenible.

Se han corroborado algunas de las tesis de otros autores ya que hemos podido comprobar que esta metodología de trabajo ha fomentado la motivación de los alumnos y esto se ha notado durante los meses de trabajo que ha durado la experiencia. Los alumnos han estado muy animados en todas las fases del trabajo; desde el planteamiento del problema inicial, búsqueda de soluciones mediante lluvia de ideas, primeros bocetos, proyección y diseño digital en 3D.

Los resultados de la Olimpiada fueron recogidos por los coordinadores de la misma que destacaron la capacidad del alumnado para identificar problemas y solucionarlos.

Más allá de estos números, los resultados de los trabajos presentados han sorprendido por su capacidad para identificar problemas y afrontar su solución considerando las diversas variables propias de la arquitectura. Remitiéndonos a los indicadores recogidos en la rúbrica, las propuestas han destacado por su alcance, originalidad, flexibilidad y factibilidad (Carcelén y García, 2018).

Presentación del artículo: 9 de enero de 2019

Fecha de aprobación: 5 de septiembre de 2019

Fecha de publicación: 30 de octubre de 2019

Del Cerro Velázquez, F. y Lozano Rivas, F. (2019). Proyecto Técnico Ecourbano apoyado en las TIC para el aprendizaje STEM (Dibujo Técnico) y la consolidación de los ODS en el aula. RED. Revista de Educación a Distancia, 60. DOI: http://dx.doi.org/10.6018/red/60/04

\section{Financiación}

La experiencia presentada en esta investigación recibió el fallo del tribunal como primer premio (con una financiación de 400€) de la I Olimpiada de Arquitectura de la Región de Murcia 2018. "Make Cool Your School". Convocatoria de "Ayudas a la organización de Olimpiadas Científicas de la Región de Murcia, financiada por la Consejería de Empleo, Universidades y Empresa de la CARM, a través de la Fundación Séneca.

\section{Referencias}

Alcober, J. Ruíz, S. Valero, M. (2003). Evaluación de la implantación del aprendizaje basado en proyectos en la epsc. Recuperado de: https://ocw.upc.edu/sites/all/modules/ocw/estadistiques/download.php?file=14589/2011/1/54324/40 149-3444.pdf

Arkiplus, (2017). Urbanismo ecológico. Recuperado el 28 de Marzo de 2018, de Arkiplus: http://www.arkiplus.com/urbanismo-ecologico

Ausin, V., Abella, V., Delgado, V., y Hortigüela, D., (2016). Aprendizaje Basado en Proyectos a través de las TIC. Una Experiencia de Innovación Docente desde las Aulas Universitarias. Recuperado de: https://scielo.conicyt.cl/scielo.php?pid=S0718-50062016000300005\&script=sci_arttext\&tlng=en

Batchelor, M.R., Wiebe, E. N. (1995), Teaching Three-Dimensional Computer Modeling: Past History and Future Plans. Recuperado de:

https://pdfs.semanticscholar.org/3a64/15537d66f22269db0f58952fefbca2aa349f.pdf 
Carcelén, R. y García, F.M. (2018). MAKE COOL YOUR SCHOOL. I olimpiada de Arquitectura de la Región de Murcia. Cartagena: CRAI Biblioteca - Universidad Politécnica de Cartagena.

Colás, M.P., De Pablos, J. y Ballesta, J. (2018). Incidencia de las TIC en la enseñanza en el sistema educativo español: una revisión de la investigación. Revista de Educación a Distancia (56), p.20. En: https://www.um.es/ead/red/56/colas_et_al.pdf

Cabero, J. (2010). Los retos de la integración de las TICs en los procesos educativos. Límites y posibilidades. Perspectiva Educacional (1), p.53. En: https://dialnet.unirioja.es/descarga/articulo/3579891.pdf

Chacón, E., Valero, E., y Valverde, I., (2012). Espacios de oportunidad. El reciclaje urbano en el contexto de la renovación del hábitat social en Francia. Recuperado de: http://institucional.us.es/revistas/habitat/5/N05A05\%20Espacios\%20de\%20oportunidad.pdf

Chueca Goitia, F. (2009). Breve historia del urbanismo. Madrid: Alianza Editorial.

Decreto n. ${ }^{\circ} 221 / 2015$ de 02 de septiembre de 2015 por el que se establece el currículo del Bachillerato en la Comunidad Autónoma de la Región de Murcia. Boletín Oficial de la Región de Murcia, núm. 203, 03 de septiembre de 2015, pp. 31684-31685.

Del Cerro, F. y Lozano, F. (2018). Estudio de un caso de enseñanza de materias STEM a través del ecourbanismo apoyado por herramientas avanzadas de diseño, en el horizonte 2030 de objetivos de desarrollo sostenible (ODS). Revista de Educación a Distancia (58), p.13. En: http://www.um.es/ead/red/58/cerro_et_al.pdf

Del Cerro, F. y Morales, G. (2017). Realidad Aumentada como herramienta de mejora de la inteligencia espacial en estudiantes de educación secundaria. Revista de Educación a Distancia, (53), p.11. En: https://www.um.es/ead/red/54/del_cerro_morales.pdf

Gacto, M. y Albadalejo, J.J. (2014). Reflexiones sobre la docencia del Dibujo Técnico en los niveles de Bachillerato: una propuesta metodológica basada en el Aprendizaje Cooperativo y las Nuevas Tecnologías. El Artista (11). En https://www.redalyc.org/articulo.oa?id=87432695005

García-Varcálcel Muñoz-Repiso, A. y Basilotta Gómez-Pablos, V. (2017). Aprendizaje basado en proyectos (ABP): evaluación desde la perspectiva de alumnos de Educación Primaria. Revista de Investigación Educativa, 35(1), 113-131 DOI: http://dx.doi.org/10.6018/rie.35.1.246811

Hertzberger, H. (2008). Space and learning. Rotterdam.

Jiménez, A.M. (2009). La escuela nueva y los espacios para educar. Revista Educación y Pedagogía, (54), pp. 103-125.

Ley Orgánica 8/2013, de 9 de diciembre, para la mejora de la calidad educativa. Boletín Oficial del Estado, núm. 295, 10 de diciembre de 2013.

Lowenfeld, V. y Brittain, W. L. (2008). Desarrollo de la capacidad intelectual y creativa. Madrid: Síntesis, S.A.

Marqués, P. (2012). Impacto de las TIC en la educación: Funciones y Limitaciones. ${ }^{3}$ Ciencias. En https://www.3ciencias.com/wp-content/uploads/2013/01/impacto-de-las-tic.pdf

Martínez, R. y Ruíz, A. (2018). Espacios para aprender. Arquitectura y docencia. Recuperado de: https://veredes.es/blog/espacios-para-aprender-arquitectura-y-docencia-i-raquel-martinez-albertoruizl

Naciones Unidas. (2019). Objetivo 4: Garantizar una educación inclusiva, equitativa y de calidad y promover oportunidades de aprendizaje durante toda la vida para todos. Recuperado de: https://www.un.org/sustainabledevelopment/es/education/

Navarro, R., Saorín, J. L., Contero, M. y Conesa, J. (2004). El dibujo del croquis y la visión espacial: su aprendizaje y valoración en la formación del ingeniero a través de las nuevas tecnologías. Ponencia presentada en XII Congreso Internacional de Innovación Educativa. Recuperado de: http://www.regeo.uji.es/publicaciones/NSCC04.pdf

OCDE. (2017), Marco de Evaluación y de Análisis de PISA para el Desarrollo: Lectura, matemáticas y ciencias, Versión preliminar, OECD Publishing, Paris. 
Pisa, M. y Novejarque, J, (2017). Las TICs en el aula y su efecto final en el resultado de aprendizaje. Ponencia presentada en el IV Congreso Internacional sobre Aprendizaje, Innovación y Competitividad. Recuperado de: https://zaguan.unizar.es/record/62974/files/148.pdf

Santos, T., Almeida, M., Bragança, L. y Barbosa, M.T. (2019). La inclusión de un indicador de conciencia de sostenibilidad en herramientas de evaluación para edificios de escuelas secundarias. Sostenibilidad, 11 (2), 387. doi: 10.3390 / su11020387

UNESCO. (2009). Medición de las tecnologías de la información y la comunicación (TIC) en educación - Manual de usuario. Recuperado de: https://unesdoc.unesco.org/ark:/48223/pf0000188309

UNESCO. (2015). Report of the International Conference on ICT and Post-2015 Education. Recuperado de: https://unesdoc.unesco.org/ark:/48223/pf0000243076

UNESCO. (2017). 2017 Declaración de Qingdao. Estrategias de movilización de las TIC para realizar la agenda Educación 2030.Recuperado de: https://unesdoc.unesco.org/ark:/48223/pf0000253061

UNESCO. (2018). Aprovechar las TIC para alcanzar las metas de Educación 2030. Recuperado de: https://es.unesco.org/themes/tic-educacion/weidong 